\title{
Experimental signature of phase-coherent Andreev reflection
}

\author{
K.-M. H. Lenssen* \\ Department of Applied Physics, Delft University of Technology, P.O. Box 5046, NL-2600 GA Delft, The Netherlands \\ M. R. Leys and J. H. Wolter \\ Department of Applied Physics, Eindhoven University of Technology, P.O. Box 513, NL-5600 MB Eindhoven, The Netherlands
}

(Received 21 January 1998)

\begin{abstract}
We report experimental observation of a signature of phase-coherent Andreev reflection. In highly transmissive, superconducting contacts to the high mobility two-dimensional electron gas in $\mathrm{GaAs}_{\mathrm{Al}} \mathrm{Al}_{x} \mathrm{Ga}_{1-x} \mathrm{As}$ heterostructures, a sharp conductance dip is found around zero bias voltage, which is ascribed to an enhancement of weak localization by Andreev reflection. This phenomenon is suppressed not only by increased temperature, a small magnetic field, or a large bias current, but also by applying a large negative voltage to a nearby gate. This behavior is in agreement with recent theoretical predictions. [S0163-1829(98)05731-2]
\end{abstract}

Interference effects in normal metals or semiconductors will be influenced dramatically if a superconductor is brought into the vicinity. Due to Andreev reflection, ${ }^{1}$ an electron can be retroreflected as a hole at the normal metalsuperconductor (NS) interface (and vice versa). In particular, within a distance of the phase coherence length $l_{\varphi}$ from the superconductor this will have large consequences for the electronic transport properties.

One of the earliest experimental observations of phasecoherent Andreev reflection was done a few years ago. ${ }^{2}$ In the $G-V$ curve of a Nb/ $\operatorname{In}_{x} \mathrm{Ga}_{1-x}$ As junction a sharp conductance peak was found around zero bias. This feature was described in terms of phase-coherent Andreev reflection. ${ }^{3-5}$ Later, this phenomenon, now known as reflectionless tunneling, has also been observed in other materials. ${ }^{6-9}$ It was realized $^{4,5}$ that other interference effects can be expected if mesoscopic systems are combined with superconductors. In this paper we will discuss the experimental observation of such a phenomenon: the enhancement of weak localization.

Semiconductors have the advantage above metals that the electron density can be modulated by means of gate structures. Especially in mesoscopic physics, nanostructures are often fabricated by means of fine gate patterns. ${ }^{10}$ It is obviously a big advantage if this valuable experience in submicrometer technology can be used for the study of phasecoherent Andreev reflection. Therefore, we developed a process for making diffused superconducting contacts to the two-dimensional electron gas (2DEG) in $\mathrm{GaAs} / \mathrm{Al}_{x} \mathrm{Ga}_{1-x} \mathrm{As}$ heterostructures. ${ }^{11}$ Clear indications of Andreev reflection have been found in these contacts and the transmission of the NS interface turned out to be close to unity. ${ }^{12}$

We used this process to make a sample with a quantum point contact between two $10-\mu \mathrm{m}$-wide superconducting contacts at a mutual distance of $\approx 0.8 \mu \mathrm{m}$ (see the inset of Fig. 1). The $2 \mathrm{DEG}$ has an electron density $n_{e} \approx 2.4$ $\times 10^{15} \mathrm{~m}^{-2}$ and a mobility $\mu \approx 110 \mathrm{~m}^{2} / \mathrm{V}$ s. This yields an elastic mean free path $l_{e} \approx 9 \mu \mathrm{m}$. The measurements are performed in a dilution refrigerator with a base temperature of $\approx 10 \mathrm{mK}$, using a current-biased lock-in technique.

Measurements of the conductance of this sample as a function of the voltage $V_{g}$ on the gates that form the quan- tum point contact have shown conductance quantization in steps larger than $2 e^{2} / h,{ }^{13}$ providing evidence for Andreev reflection. From the observation that the quantization unit is approximately $2.2 e^{2} / h$ instead of $4 e^{2} / h$ (which is expected for the ideal, clean case) we can make a rough estimation of the effective transmission probability of the sample. Under the assumption that the transmission $T$ is mode independent, we find an effective value of 0.85 , using the generalization of the Landauer-Büttiker formula for an NS junction ${ }^{4}$

$$
G_{\mathrm{NS}}=\frac{4 e^{2}}{h} \sum_{n=1}^{N} \frac{T_{n}^{2}}{\left(2-T_{n}\right)^{2}} .
$$

This effective transmission value is also consistent with an analogous estimation using the Landauer-Büttiker formula in the normal state. We think that the transmission is reduced due to disorder (induced by the diffusion process) near the NS interface and a nonunity transmission of the interface itself. Anyway, this argument shows that the transmission of an eventual barrier at the NS interface is larger than $\approx 0.85$, which is clearly in the regime where enhanced weak localization effects can be expected, according to Ref. 5 .

In Fig. 1 the differential conductance of the sample is shown as a function of the bias voltage at zero gate voltage $V_{g}$. Without magnetic field a broad minimum with a sharp dip is observed around zero bias voltage. A similar $G-V$ curve is also found for contacts that are $470 \mu \mathrm{m}$ apart, so this phenomenon must be related to separate NS interfaces and not to a coupled SNS system. If the magnetic field is increased, the whole feature vanishes at a value well below 60 $\mathrm{mT}$.

The effect of increasing temperature can be seen in Fig. 2: After a rapid rounding of the minimum the whole structure has disappeared at $4.2 \mathrm{~K}$. Detailed inspection of the lowtemperature curve (without magnetic field) suggests that the minimum consists of two superimposed phenomenon. This suggestion is confirmed by measurements on a sample where the major part of the gates (black in the inset of Fig. 1) between the two small superconducting contacts does not function, but the current path still can be confined by the wider gates besides the small contacts (gray in the inset). The 


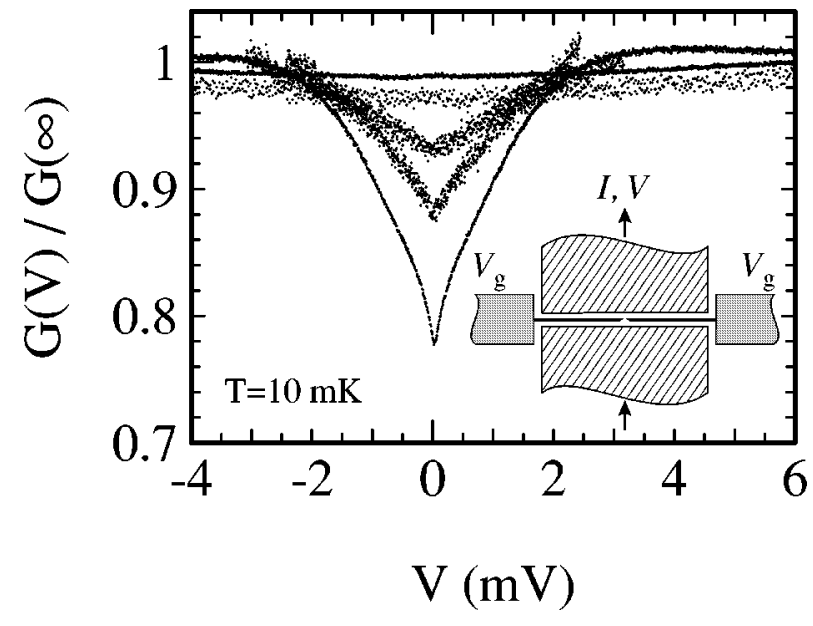

FIG. 1. Normalized differential conductance as a function of the bias voltage at several magnetic fields $\left(V_{g}=0 \mathrm{mV}\right.$; from top to bottom $B=60,35,20,15$, and $0 \mathrm{mT}$ ); the inset shows the layout of the center of the sample on scale (the hatched areas are Ti/Sn contacts, the gray and black areas are gold gates).

conductance of this sample is presented in Fig. 3. At $T$ $=0.9 \mathrm{~K}$ only a broad minimum is observed, while at lower temperatures a sharp conductance dip is superimposed on this. This clearly shows the two constituents of the effect. In Fig. 2 we see that at $T=0.9 \mathrm{~K}$ the conductance minimum has become much more rounded than at lower temperatures, while the rest of the curve has barely changed, which is in accordance with the concept of two separate effects.

The broad conductance minimum can be well described by Andreev reflection at a nonideal NS interface. ${ }^{14}$ If the interface has a finite transmission, a reduction in the conductance is expected below the superconducting gap, due to the fact that Andreev reflection is a two-particle process, in contrast to electron tunneling, which is a one-particle process. The value of the temperature and magnetic field at which the minimum is suppressed are in reasonable agreement with the superconducting properties of tin $\left(T_{c 0}=3.7 \mathrm{~K}\right.$ and $B_{c 0}=30$ $\mathrm{mT}$ for bulk $\mathrm{Sn}$ ). That the bias voltage at which the conductance reduction in our measurements starts is not exactly

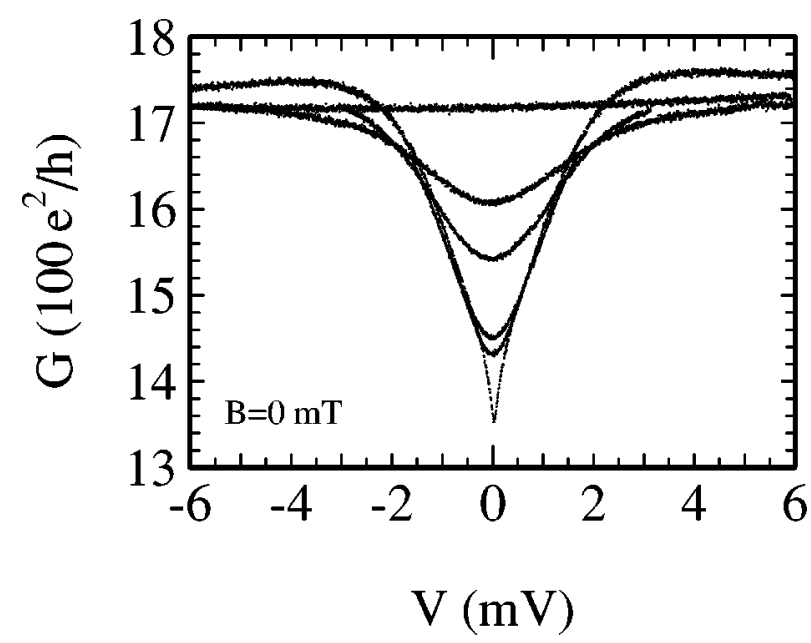

FIG. 2. Differential conductance as a function of the bias voltage at several temperatures $\left(V_{g}=0 \mathrm{mV}\right.$; from top to bottom $T$ $=4.2,2.5,1.8,0.9,0.75$, and $0.01 \mathrm{~K})$.

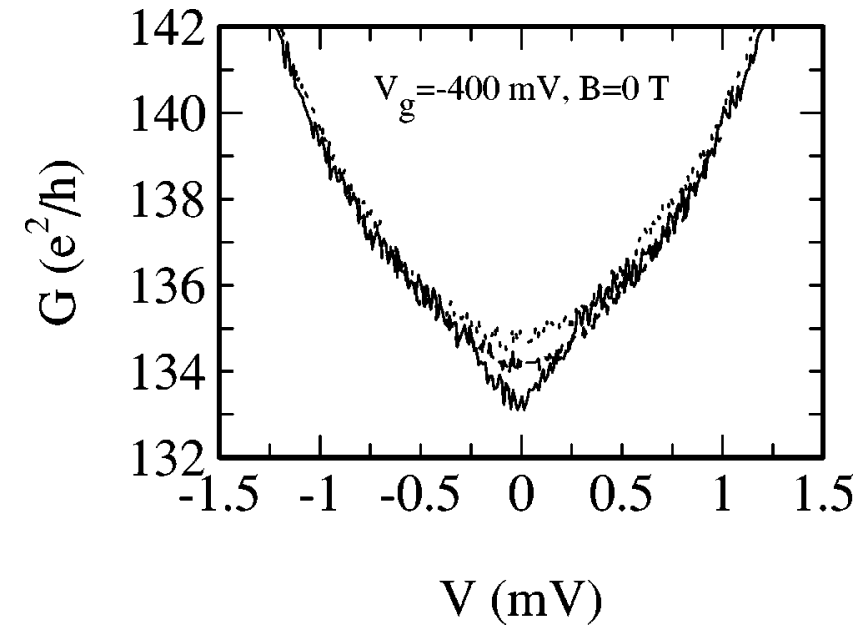

FIG. 3. Differential conductance as a function of the bias voltage at several temperatures (without quantum point contact; $V_{g}=$ $-400 \mathrm{mV}$; from top to bottom $T=0.9,0.7$, and $0.01 \mathrm{~K}$ ).

equal to twice the superconducting gap $\Delta$ of tin can be explained by a series resistance of $\approx 10 \Omega$. Of course it is also possible that the superconducting properties have been changed by impurities or even alloys in the tin.

We attribute the small, sharp conductance dip to phasecoherent Andreev reflection. In a junction between a superconductor and a disordered normal material weak localization effects can be enhanced by Andreev reflection if the barrier at the NS interface is not too high. ${ }^{4,5}$ It is as if Andreev reflection effectively doubles the length of the disordered region. In contrast to conventional weak localization effects, this phenomenon changes the (low) $V$ dependence of the conductance: Around zero bias the conductance will be reduced, but at larger bias voltages phase coherence between the electrons and Andreev-reflected holes is broken and the conductance approaches again its "normal" value. This is what we see in Figs. 2 and 3; The sharp dip is suppressed at a critical voltage $V_{c} \approx 0.25 \mathrm{mV}$. Evidently, weak localization effects can be suppressed by a small magnetic field, since then time reversal symmetry is broken. This is also observed in the measurements (Figs. 1 and 4). The conductance first increases as a function of the magnetic field, because weak

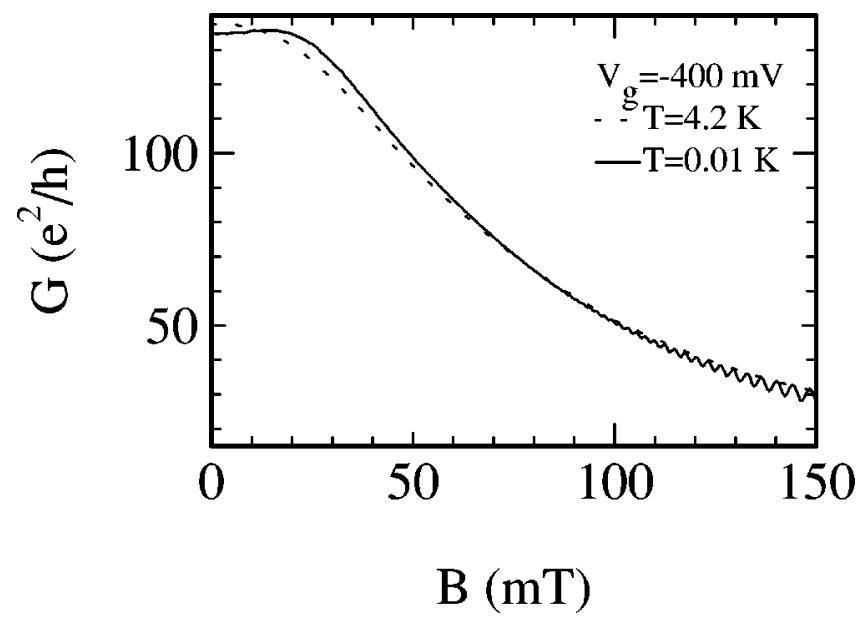

FIG. 4. Differential conductance as a function of the magnetic field (without quantum point contact; $V_{g}=-400 \mathrm{mV}$ ). 
localization is suppressed, before it decreases due to the square magnetoresistance of the 2DEG. ${ }^{15}$ This is in contrast to the monotonic behavior at elevated temperature. In $G-V$ curves in magnetic fields it is noticed that at the same $B$ value the small conductance dip has practically vanished.

Theoretically, ${ }^{5}$ the critical magnetic field for the weak localization is given by

$$
B_{c}=\frac{h}{e l_{\varphi}^{2}}
$$

if the phase coherence length is smaller than the length of the disordered normal region. From this equation we estimate $l_{\varphi}$ to be of the order of $0.4 \mu \mathrm{m}$. A quantitative prediction of $V_{c}$ by means of the expression

$$
e V_{c}=\frac{\pi}{2} \frac{\hbar v_{F} l_{e}}{l_{\varphi}^{2}}
$$

is difficult since the Fermi velocity $v_{F}$ and the mean free path $l_{e}$ of the disordered region are not known. However, the experimentally found $V_{c}$ and $T_{c}$ should be related by $e V_{c}$ $\approx 4 k_{B} T_{c}$. From $V_{c} \approx 0.25 \mathrm{mV}$ we would expect $T_{c} \approx 0.75 \mathrm{~K}$. This is in agreement with the measurements. Moreover, the absolute depth of the conductance dip can be estimated theoretically. Indeed, the experimental observation for $V_{g}=$ $-400 \mathrm{mV}$, where the current path is confined within the 2DEG region between the two superconducting contacts (by the gray gates in the inset), has the right order of magnitude. Since both the length $L$ and the width $W$ of the relevant region are larger than $l_{\varphi}$ and there are two NS interfaces, a dip of order $2\left(W / l_{\varphi}\right)\left(l_{\varphi} / L\right)^{2} \times 0.5 e^{2} / h \approx 6 e^{2} / h$ would be expected. ${ }^{16}$ This is about three times as large as experimentally observed in Fig. 3, but nonideality of the NS interfaces might easily account for this discrepancy. ${ }^{17}$

The temperature dependence of the normalized differential conductance change is plotted in Fig. 5. At low temperatures and without magnetic field applied the conductance is increasing with temperature. If the enhanced weak localization is suppressed, a very different dependence is observed; even the sign of the conductance change is opposite. As can be seen in the graph this suppression can be achieved by applying a small magnetic field or a large dc. ${ }^{18}$ The most important observation, however, is the fact that the enhanced weak localization can also be suppressed by a large negative voltage on the nearby gates of the quantum point contact (black in the inset). This proves that phase coherence is preserved down to in the 2DEG since the electrical properties of

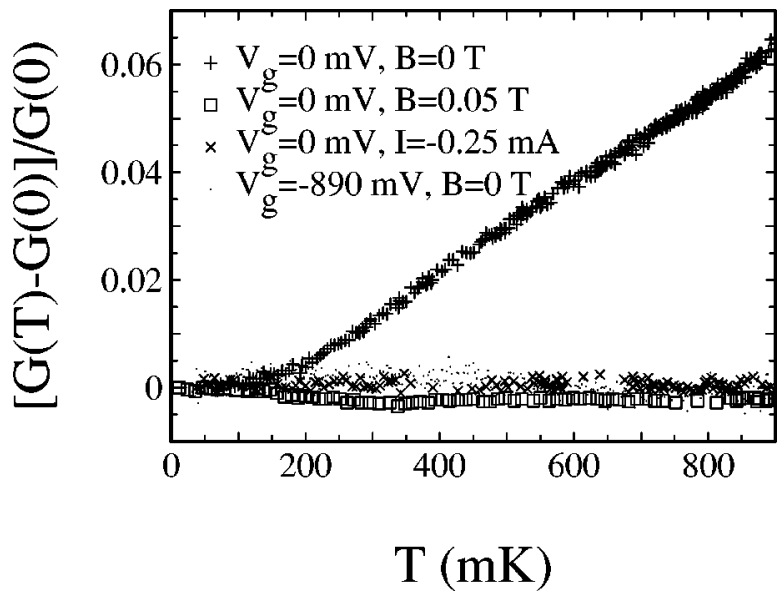

FIG. 5. Temperature dependence of the normalized conductance change.

metals (like the contacts themselves) cannot be affected by a voltage on the gates. This is also predicted by the estimation of $l_{\varphi} \approx 0.4 \mu \mathrm{m}$, which is much larger than the distance of the 2DEG from the surface $(\approx 85 \mathrm{~nm})$ and, evidently, also follows from the observed conductance quantization in enhanced units. We think that the explanation for the suppression is the formation of a potential barrier in the quantum point contact. This lowers the total transmission of the sample, which is then not in the regime of enhanced weak localization anymore, but rather in the regime of reflectionless tunneling. ${ }^{5}$ Indeed, we have observed a maximum in the $G-V$ curve at zero bias for $V_{g}=-890 \mathrm{mV}$, instead of the minimum found for $V_{g}=0 \mathrm{mV}$.

In summary, we have presented the experimental observation of the enhancement of weak localization by Andreev reflection. In correspondence with theoretical predictions, the effect is suppressed by increasing temperature, a small magnetic field, or a large dc. Moreover, the enhancement vanishes when a large negative voltage is applied to the nearby quantum point contact gates. This proves that phase coherence between Andreev-reflected particles exists in the 2DEG.

We are very grateful to C. J. P. M. Harmans, J. E. Mooij, and C. W. J. Beenakker for helpful discussions, to L. A. Westerling and P. C. A. Jeekel for help with the experiments and analysis of the data, and to the Delft Institute of MicroElectronics and Submicron Technology (DIMES) for the use of their facilities. This research was financially supported by the Dutch Foundation for Fundamental Research on Matter (Stichting FOM).
*Present address: Philips Research Laboratories, Prof. Holstlaan 4, NL-5656 AA Eindhoven, The Netherlands.

${ }^{1}$ A. F. Andreev, Zh. Éksp. Teor. Fiz. 46, 1823 (1964) [Sov. Phys. JETP 19, 1228 (1964)]; 49, 655 (1965) [ 22, 455 (1965)].

${ }^{2}$ A. Kastalsky, A. W. Kleinsasser, L. H. Greene, R. Bhat, F. P. Milliken, and J. P. Harbison, Phys. Rev. Lett. 67, 3026 (1991).

${ }^{3}$ B. J. van Wees, P. de Vries, P. Magnée, and T. M. Klapwijk, Phys. Rev. Lett. 69, 510 (1992).

${ }^{4}$ C. W. J. Beenakker, Phys. Rev. B 46, 12841 (1992).

${ }^{5}$ I. K. Marmorkos, C. W. J. Beenakker, and R. A. Jalabert, Phys. Rev. B 48, 2811 (1993).
${ }^{6}$ C. Nguyen, H. Kroemer, and E. L. Hu, Phys. Rev. Lett. 69, 2847 (1992).

${ }^{7}$ K.-M. H. Lenssen, L. A. Westerling, P. C. A. Jeekel, C. J. P. M. Harmans, J. E. Mooij, M. R. Leys, W. van der Vleuten, J. H. Wolter, and S. P. Beaumont, Physica B 194-196, 2413 (1994).

${ }^{8}$ P. H. C. Magnée, N. van der Post, B. J. van Wees, and T. M. Klapwijk, Physica B 194-196, 1031 (1994).

${ }^{9}$ S. J. M. Bakker, E. van der Drift, T. M. Klapwijk, H. M. Jaeger, and S. Radelaar, Phys. Rev. B 49, 13275 (1994).

${ }^{10}$ See, for example, C. W. J. Beenakker and H. van Houten, Solid State Phys. 44, 1 (1991), and references therein. 
${ }^{11}$ K.-M. H. Lenssen, M. Matters, C. J. P. M. Harmans, J. E. Mooij, M. R. Leys, W. van der Vleuten, and J. H. Wolter, IEEE Trans. Appl. Supercond. 3, 1961 (1993).

${ }^{12}$ K.-M. H. Lenssen, M. Matters, C. J. P. M. Harmans, J. E. Mooij, M. R. Leys, W. van der Vleuten, and J. H. Wolter, Appl. Phys. Lett. 63, 2079 (1993).

${ }^{13}$ K.-M. H. Lenssen, L. A. Westerling, C. J. P. M. Harmans, J. E. Mooij, M. R. Leys, W. van der Vleuten, and J. H. Wolter, Surf. Sci. 305, 476 (1994).

${ }^{14}$ G. E. Blonder, M. Tinkham, and T. M. Klapwijk, Phys. Rev. B 25, 4515 (1982).

${ }^{15}$ The oscillations at higher $B$ are due to the Shubnikov-de Haas effect and confirm the "normal" behavior of the 2DEG between the contacts.

${ }^{16}$ C. W. J. Beenakker (private communication).

${ }^{17}$ In the rough measurement data at $V_{g}=0 \mathrm{mV}$ the depth of the sharp dip seems to be considerably larger than $e^{2} / h$ (Fig. 2); this is probably due to the many parallel paths between the superconducting contacts that the current can follow (i.e., $W \gg l_{\varphi}$ ).

${ }^{18}$ Since the experiment is current biased, a large dc is applied. Physically, however, Andreev reflection (and thus the enhanced weak localization) is suppressed because the resulting voltage over the NS interface is much higher than the superconducting gap $\Delta$. 